\title{
On the Gas Dynamic Features of the Interacting Binary System UU Cas
}

\author{
Dmitry A. Kononov ${ }^{1}$ (D), Stanislav Yu. Gorda ${ }^{2}$, and Sergey Yu. Parfenov ${ }^{2}$ \\ ${ }^{1}$ Institute of Astronomy of the RAS, 48 Pyatnitskaya Str., Moscow, 119017, Russia; dkononov@inasan.ru, dmitry.kononov@gmail.com \\ ${ }^{2}$ Ural Federal University, 51 Lenin Str., Ekaterinburg, 620000, Russia \\ Received 2018 September 17; revised 2019 August 9; accepted 2019 August 12; published 2019 October 3
}

\begin{abstract}
We present the results of spectroscopic observations and $\mathrm{H} \alpha$ Doppler tomography of the massive close binary system UU Cas where a giant star fills its Roche lobe (donor) and transfers material through the vicinity of the $L_{1}$ point onto a massive but rather compact stellar companion (accretor). The system has been observed at the $1.2 \mathrm{~m}$ telescope of the Kourovka Astronomical Observatory. By analyzing the obtained spectra and computed Doppler tomogram, we suggest that at least three elements of the gas dynamic pattern in the system contribute to the Balmer $\mathrm{H} \alpha$ emission line profiles. These elements are: the stream from the $L_{1}$ point, the stellar wind from the accretor, and the diffuse disk, surrounding the accretor. In combination with the estimates of the companions' masses, our findings indicate that the system is at the late stage of the first mass transfer phase of its evolution.
\end{abstract}

Key words: binaries: close - techniques: spectroscopic

\section{Introduction}

Despite the discrepancies in the estimation of orbital parameters, it is generally accepted that UU Cas is a massive close binary system. Such systems are of particular interest since, among other things, they are believed to contain supernova progenitors and future black hole and neutron star candidates (see, e.g., Podsiadlowski et al. 1992; Wellstein \& Langer 1999; Postnov \& Yungelson 2014).

Back in 1934, Sanford (Sanford 1934) obtained photographic spectrophotometry of UU Cas $\left(V=10^{\mathrm{m}} \cdot 4-10^{\mathrm{m}} .8\right.$, $P=8.51929)$ and found that it is a close eclipsing binary system containing massive components (approx. $30 M_{\odot}$, each) of early spectral types, with the brighter companion having a larger mass. Antokhina \& Kumsiashvili (1992) and Polushina (2002) have obtained similar estimates by analyzing photometric data on this object.

Photoelectric light curves of UU Cas obtained by Kumsiashvili \& Chargeishvili (2009) and Polushina (2002) have shown significant (up to 0.08 ) light variations about the mean values, prompting the authors to suppose that one of the companions (or both) fills its Roche lobe, which drives the overflow and the formation of various gaseous structures in the system.

Whereas there have been a number of works on photometric observations of the UU Cas, no detailed spectroscopic studies of the star had been performed since Sanford's work until 2010. The only exception was the study of a Galactic field area in Cassiopeia performed in the early $1970 \mathrm{~s}$ by Martin (1972), who estimated the spectral type of UU Cas as B0.5III from lowdispersion photographic spectra. The next spectroscopic study of UU Cas was initiated by Markov and colleagues in 2008 at the $2 \mathrm{~m}$ telescope of the Bulgarian National Observatory (Markov et al. 2010, 2011). They obtained spectra where the $\mathrm{H} \alpha$ line demonstrated strong emission components while the absorption He I lines showed a bimodal structure, with one of the line components significantly deeper than the other, especially at orbital phases close to $\phi=0.25$ and $\phi=0.75 .^{3}$ In addition, those spectra demonstrated weaker photospheric metal lines that,

\footnotetext{
3 Please note that Antokhina \& Kumsiashvili (1992), Polushina (2002), and Markov et al. $(2010,2011)$ start counting the orbital phases from the deeper minimum $(\phi=0)$ in the light curve.
}

however, had a unimodal structure, i.e., belonged to only one of the companions. By using these metal lines, the authors constructed velocity curves that appeared to show a semiamplitude of $25 \%$, greater than that found by Sanford (1934), but were in a good agreement with the velocity curve of the deeper He I line component. Any attempts to construct the velocity curve of the shallower He I component failed, since the authors could not obtain enough spectra at phases, where this line component was convincingly detected. By analyzing the shape of the $\mathrm{H} \alpha$ emission line, Markov et al. $(2010,2011)$ suggested that one of the binary components barely visible in the spectrum is surrounded by a thick accretion disk that may significantly absorb the flux of the star. Djurašević et al. (2010) developed a model of the UU Cas system with an accretion disk surrounding the fainter companion.

In 2017, Gorda (2017) conducted a detailed spectroscopic study of UU Cas. The data he obtained allowed for the construction of the first velocity curves for both of the components of the binary by using the absorption $\mathrm{He} \mathrm{I}$ and $\mathrm{N}$ II lines. The estimated masses of the companions appeared to be essentially lower than those obtained by Sanford (1934), Kumsiashvili \& Chargeishvili (2009), and Polushina (2002). In addition, the mass ratio appears to be opposite of that obtained from the photometric study, i.e., the mass of the brighter companion filling its Roche lobe is half the mass of the compact companion $^{4} \quad\left(M_{1} \sin ^{3}(i)=14.4 \pm 2.3 M_{\odot} \quad\right.$ and $\quad M_{2} \sin ^{3}(i)=$ $\left.7.7 \pm 2.3 M_{\odot}\right)$, which essentially modifies our view of the evolutionary status of UU Cas. The recent data show that the system is, supposedly, in the late stage of the first mass transfer. Here arises a fact seemingly paradoxical at a glance. This fact is that the deeper minimum is observed when the more massive and, hence, hotter primary star eclipses the cooler secondary. However, this paradox can be solved within the above mentioned model by Djurašević et al. (2010) where the primary is surrounded by the disk that may effectively occult the secondary.

\footnotetext{
4 Hereafter, contrary to the "observational" notation, where the "primary" is the brighter companion, we use the "physical" notation assuming the primary to be the more massive companion. We also start counting orbital phases from the moment when the low-mass (but brighter) companion filling its Roch lobe eclipses the compact massive star. In our case, this means that $\phi=0$ corresponds to the shallower minimum in the light curve.
} 
Table 1

New Spectra of UU Cas Obtained from 2017 July to 2018 March

\begin{tabular}{lrcc}
\hline \hline Date & HJD-2,458,000 & $\begin{array}{c}\text { Total Exp. Time } \\
(\mathrm{hr})\end{array}$ & Phase \\
\hline 2017 Jul 22 & -037.68159 & 3.0 & 0.2644 \\
2017 Nov 10 & 038.15110 & 2.0 & 0.1657 \\
2018 Jan 5 & 124.27512 & 3.0 & 0.2738 \\
2018 Jan 6 & 125.22942 & 2.0 & 0.3797 \\
2018 Jan 12 & 131.16566 & 3.0 & 0.0838 \\
2018 Feb 8 & 158.23150 & 2.0 & 0.2608 \\
2018 Feb 9 & 159.14144 & 3.0 & 0.3677 \\
2018 Feb 16 & 166.15118 & 2.5 & 0.1909 \\
2018 Mar 19 & 197.18453 & 1.5 & 0.8332 \\
\hline
\end{tabular}

As, for instance, Podsiadlowski et al. (1992) and Postnov \& Yungelson (2014) mentioned in their works, the final product of the binary evolution strongly depends on the type of interaction between the companions, in particular, the character of the mass exchange process. Therefore, it is important, inter alia, to investigate the gas dynamic pattern in a system and reveal the details of the mass transfer process to understand its evolutionary state and further evolutionary path. In this paper, we continue the study initiated by Gorda (2017) and focus on the gas dynamic features of the UU Cas system, since these features significantly contribute to the observational data, both photometric and spectroscopic, and may give information on system's evolutionary scenario. Herein, we use spectral data and Doppler tomography to describe the UU Cas flow pattern in detail.

The paper is organized as follows. In Section 2, we describe the observations and data reduction procedures. In Section 3, we analyze the obtained spectral data and estimate the parameters of the companions. Section 4 is focused on the analysis of $\mathrm{H} \alpha$ emission line profiles and a proposed phenomenological model of the flow pattern in the system. Section 5 is devoted to Doppler tomography of UU Cas. In Section 7, we summarize our results and discuss possible future studies of the system.

\section{Observations, Data Reduction, and Spectral Analysis}

In this study, we use the spectra obtained by Gorda (2017) 2017 January-April as well as new spectra obtained 2017 July through 2018 March. A summary of the observational data is given in Table 1 where the phase has been calculated from the following equation (Gorda 2017), taking into account that we utilize the phases shifted by 0.5 from those in Gorda (2017):

$$
\mathrm{JD}_{\odot}=2445726 \text { d. } 365215+8.51929 \mathrm{E},
$$

with $\mathrm{E}$ denoting the number of the cycles. The reported observations were carried out with the fiber-fed high-resolution $(R=15,000)$ echelle spectrograph (Panchuk et al. 2011; Krushinsky et al. 2014) mounted on the $1.2 \mathrm{~m}$ telescope of The Kourovka Astronomical Observatory of the Ural Federal University (Ekaterinburg, Russia).

Each individual frame contains 60 overlapping spectral orders and covers the wavelength range of 4100-7500 $\AA$. In order to construct the dispersion curves, we used the spectrum of a Th-Ar lamp obtained before and after each series of the 30 minute exposures of the object. The nightly series of frames of the UU Cas spectrum were median stacked to increase the signal-to-noise ratio $(\mathrm{S} / \mathrm{N})$ and remove cosmic rays. The effective exposures of the median stacked frames range from one to three hours.
Table 2

Difference in Abundances Used by Lanz \& Hubeny (2007) for Calculations of the BSTAR2006 Grid of Theoretical Stellar Models with Solar Chemical Composition and Chemical Composition Appropriate for Supergiants

\begin{tabular}{lcc}
\hline \hline Elemental Abundance & Solar Models & Supergiant Models \\
\hline $\mathrm{He} / \mathrm{H}$ & 0.1 & 0.2 \\
$\mathrm{C} / \mathrm{H}$ & $3.31 \times 10^{-4}$ & $1.65 \times 10^{-4}$ \\
$\mathrm{~N} / \mathrm{H}$ & $8.32 \times 10^{-5}$ & $4.16 \times 10^{-4}$ \\
\hline
\end{tabular}

Note. The abundances of the other 27 elements in the supergiant models are the same as those in the solar models and are from Grevesse \& Sauval (1998).

We reduced the raw data by using the $\mathrm{DECH}^{5}$ software package. The reduction procedure included the standard steps of bias subtraction, flat-fielding, sky background subtraction, echelle order extraction, and wavelength calibration. The last step was the continuum normalization.

\section{Fitting Balmer Photospheric Absorption Line Profiles}

Extraction of the emission component of the $\mathrm{H} \alpha$ line tracing gas dynamic features in the system requires the subtraction of the underlying absorption photospheric component from the observed spectra. The profile of this absorption component was modeled using theoretical spectra calculated for both the companions. We stress that our main aim was to estimate the parameters of theoretical spectra that allow to fit best the Balmer absorption line profiles. These profiles depend mainly on the effective temperature ( $\left.T_{\text {eff }}\right)$ and surface gravity $(\log g$ ) and are not very sensitive to the chemical composition and microturbulent velocity, $v_{t}$ (see, e.g., Nieva \& Przybilla 2010).

The theoretical spectra were obtained from the BSTAR2006 grid of theoretical stellar spectra (Lanz \& Hubeny 2007) calculated for a range of $T_{\text {eff }}$ and $\log g$. The BSTAR2006 grid includes the spectra calculated for three sets of chemical composition and $v_{t}$ : (1) the solar chemical composition and $v_{t}=2 \mathrm{~km} \mathrm{~s}^{-1}$; (2) solar chemical composition and $v_{t}=$ $10 \mathrm{~km} \mathrm{~s}^{-1}$; (3) supergiant chemical composition (see Table 2) and $v_{t}=10 \mathrm{~km} \mathrm{~s}^{-1}$. We then convolved these spectra with the instrumental and rotational profiles for given values of projected spin rotational velocity $V$ sin $i$ using ROTIN3 program distributed with the TLUSTY software package (Hubeny \& Lanz 1995). The model profiles do not account for the tidal deformation of the secondary nor any gravitational darkening. Neither do they account for any mutual occultations around the eclipse phases.

To calculate the theoretical spectrum of the binary, we used the approach similar to that of Tkachenko (2015) involving the ratio $q_{R}=\left(R_{2} / R_{1}\right)^{2}$, where $R_{1}$ and $R_{2}$ are the primary and secondary companions' radii, respectively. The study of Polushina (2002) shows that there is also a third light, which is probably related to the circumstellar gas. Since we had information on neither the circumstellar gas properties nor the photometric data obtained simultaneously with our spectra, we modeled the contribution of the third light in a simple manner as a wavelength independent fraction, $l_{3}$, of the binary continuum. With $q_{R}$ and $l_{3}$, the theoretical normalized spectrum

\footnotetext{
5 http://www.gazinur.com/DECH-software.html
} 
of the binary can be calculated as

$$
I t=\frac{I_{1}+q_{R} I_{2}+l_{3}\left(I_{1}^{c}+q_{R} I_{2}^{c}\right)}{\left(I_{1}^{c}+q_{R} I_{2}^{c}\right)\left(1+l_{3}\right)},
$$

where $I_{i}$ is the total intensity in the theoretical spectrum of the $i$ th binary component and $I_{i}^{c}$ is the continuum intensity of the same companion. The brightness variations, which can be related to the third light, do not exceed $10 \%$. We have chosen a somewhat wider range for the third light contribution of $l_{3}= \pm 0.3$. To take into account the uncertainty of the binary velocity curve, we varied the radial velocity shifts of theoretical spectra by $\Delta V$ with respect to the velocity curve constructed by Gorda (2017). We varied $\Delta V$ from -10 to $10 \mathrm{~km} \mathrm{~s}^{-1}$, which is consistent with the rms deviation of $\sim 10 \mathrm{~km} \mathrm{~s}^{-1}$ of the line velocity shifts from the velocity curve estimated by Gorda (2017). We, therefore, calculated the radial velocities of the primary, $V_{\mathrm{r} 1}$, and secondary, $V_{\mathrm{r} 2}$, as

$$
\begin{aligned}
& V_{\mathrm{r} 1}(\phi)=-36.2-102.2 \sin (2 \pi \phi)+\Delta V_{1}, \\
& V_{\mathrm{r} 2}(\phi)=-60.7+190.3 \sin (2 \pi \phi)+\Delta V_{2} .
\end{aligned}
$$

To estimate the parameters of the theoretical spectra needed to fit the Balmer absorption line profiles, we used Bayesian analysis based on Bayes' theorem:

$$
P(\Theta \mid D) \propto P(D \mid \Theta) P(\Theta),
$$

where $D$ and $\Theta$ represent the data and model, respectively; $P(\Theta)$ is the prior probability; $P(D \mid \Theta)$ is the likelihood function that is the probability of the data given the model; and $P(\Theta \mid D)$ is the posterior distribution that is the probability of the model given the data. The values of $P(\Theta \mid D)$ (see Figure 1) have been calculated for the grid of parameters that are $\Theta=\left\{T_{\text {eff1 } 1}, T_{\text {eff } 2}\right.$, $\left.\log g_{1}, \log g_{2}, V \sin i_{1}, V \sin i_{2}, \Delta V_{1}, \Delta V_{2}, q_{R}, l_{3}\right\}$, where subindices of 1 and 2 denote the primary and secondary binary components, respectively.

The likelihood has been estimated from:

$$
\ln P(D \mid \Theta)=-\frac{1}{2} \frac{1}{N_{\text {lines }}} \sum_{\mathrm{i}=1}^{N_{\text {lines }}} \frac{1}{N_{\nu}^{i}} \sum_{\mathrm{j}=1}^{N_{\nu}^{i}}\left(\frac{I t_{\mathrm{j}}^{\mathrm{i}}(\Theta)-I_{\mathrm{j} \text { obs }}^{\mathrm{i}}}{\sigma^{\mathrm{i}}}\right)^{2},
$$

where $N_{\nu}^{i}$ is the number of wavelength points in the spectral line $i, N_{\text {lines }}$ is the number of spectral lines used in the analysis, $I_{\mathrm{j} \text { obs }}^{\mathrm{i}}$ is the observed normalized flux in the $j$ th point of the spectral line $i, I t_{\mathrm{j}}^{\mathrm{i}}(\Theta)$ is the synthetic normalized flux for a given set of model parameters $\Theta$, and $\sigma^{\mathrm{i}}=(\mathrm{S} / \mathrm{N})^{-1}$ accounts for the $\mathrm{S} / \mathrm{N}$ for the spectral line $i$. The $\mathrm{S} / \mathrm{N}$ has been calculated as the mean normalized flux divided by the standard deviation of the normalized flux in the continuum region close to a given line. We considered the $\mathrm{H} \gamma, \mathrm{H} \delta$ lines as well as the $\mathrm{He}$ and metal lines (lines of Si III, O II, N II, C II, Al III) shown in Figure 2.

To estimate the stellar atmosphere parameters, we used the observed line profiles in the spectrum obtained on 2018 January 5. The observed spectrum chosen for the fitting procedure has the best $\mathrm{S} / \mathrm{N}$ (up to 100) among the other spectra and corresponds to the orbital phase of 0.2738 , which ensures avoiding eclipses.

The value of $T_{\text {eff }}$ can be well estimated with the spectral lines of successive ionization stages of the same element utilizing an ionization balance (see, e.g., Martins 2011). Our observed spectra lack such lines, which can significantly complicate the analysis. We, thus, employed priors to additionally constrain the theoretical model parameters. The priors were chosen based on the binary parameters estimated by Gorda (2017) and comparing by eye the observed spectra with synthetic ones. The synthetic spectra for such a comparison were calculated for different values of $\log g$ and $q_{R}$ and for the primary and secondary $T_{\text {eff }}$ of, respectively, 30,000 and 19,000 K obtained using the BONNSAI $^{6}$ tool (Schneider et al. 2014) with the stellar mass values from Gorda (2017) as input parameters.

Comparing the observed $\mathrm{He}$ and metal line wing profiles with the synthetic ones by eye, we have chosen the Gaussian prior probabilities for $V \sin i$. The mean values of the $V \sin i$ prior distributions for the primary and secondary companions were, respectively, 150 and $100 \mathrm{~km} \mathrm{~s}^{-1}$, while the standard deviations were $50 \mathrm{~km} \mathrm{~s}^{-1}$ for both companions. We also estimated minimum and maximum $q_{R}$ values of 1 and 5, respectively. Comparing the synthetic and observed profiles of $\mathrm{N}$ II and He I lines, we found that the models with supergiant chemical composition and $v_{t}=10 \mathrm{~km} \mathrm{~s}^{-1}$ for the secondary companion and with solar chemical composition and $v_{t}=2 \mathrm{~km} \mathrm{~s}^{-1}$ for the primary companion fit the observed spectrum better than the models with other sets of chemical composition and $v_{t}$. To follow the $P(\Theta \mid D)$ calculations, we therefore used the models calculated with solar chemical composition with $v_{t}=2 \mathrm{~km} \mathrm{~s}^{-1}$ for the primary companion and supergiant chemical composition with $v_{t}=10 \mathrm{~km} \mathrm{~s}^{-1}$ for the secondary companion. We did not vary chemical composition and $v_{t}$ for calculations of $P(\Theta \mid D)$, since there is no significant variation in the Balmer line profiles between the models calculated for the different sets of chemical composition and $v_{t}$ (see Figure 3).

The prior probabilities for model parameters other than $V \sin$ $i$ were uniform for the chosen parameter ranges. The prior probabilities were set to zero for the models with the theoretical mass ratio $q_{R}^{-1}\left(g_{1} / g_{2}\right)$, which was not within the estimate $q=M_{1} / M_{2}=1.85 \pm 0.2$ from Gorda (2017). This allowed us to place constraints on $\log g$ and $q_{R}$ values and significantly reduce the computational time needed for the analysis. In total, the number of models with nonzero prior probabilities was about $1,260,000$.

The resulting marginalized posterior distributions of $T_{\text {eff }}$ and $\log g$ for both the components are rather flat (see Figure 1) and, therefore, the values of effective temperature and surface gravity cannot be well constrained. However, the spectrum obtained for the parameters having maximum posterior probability (see Table 3) generally fits well the $\mathrm{He} \mathrm{I}, \mathrm{H} \gamma$, and $\mathrm{H} \delta$ lines, and most of the metal lines; although, the model underestimates the strength of the He I $\lambda \lambda 5876,6678$ lines of the primary and secondary and the He I $\lambda 4471$ line of the secondary (Figure 2). The discrepancy for the He lines may arise because of uncertainties in the $\mathrm{He}$ abundance and $v_{t}$. Fortunately, these uncertainties insignificantly affect the theoretical profiles of the Balmer lines that we are mostly interested in. We stress that the parameters given in Table 3 should not be used for physical interpretation, as they represent only a single point from a wide range of possible parameter values. Instead, the physical interpretation should be guided by the posterior distribution given in Figure 1.

\footnotetext{
6 https://www.astro.uni-bonn.de/stars/bonnsai
} 

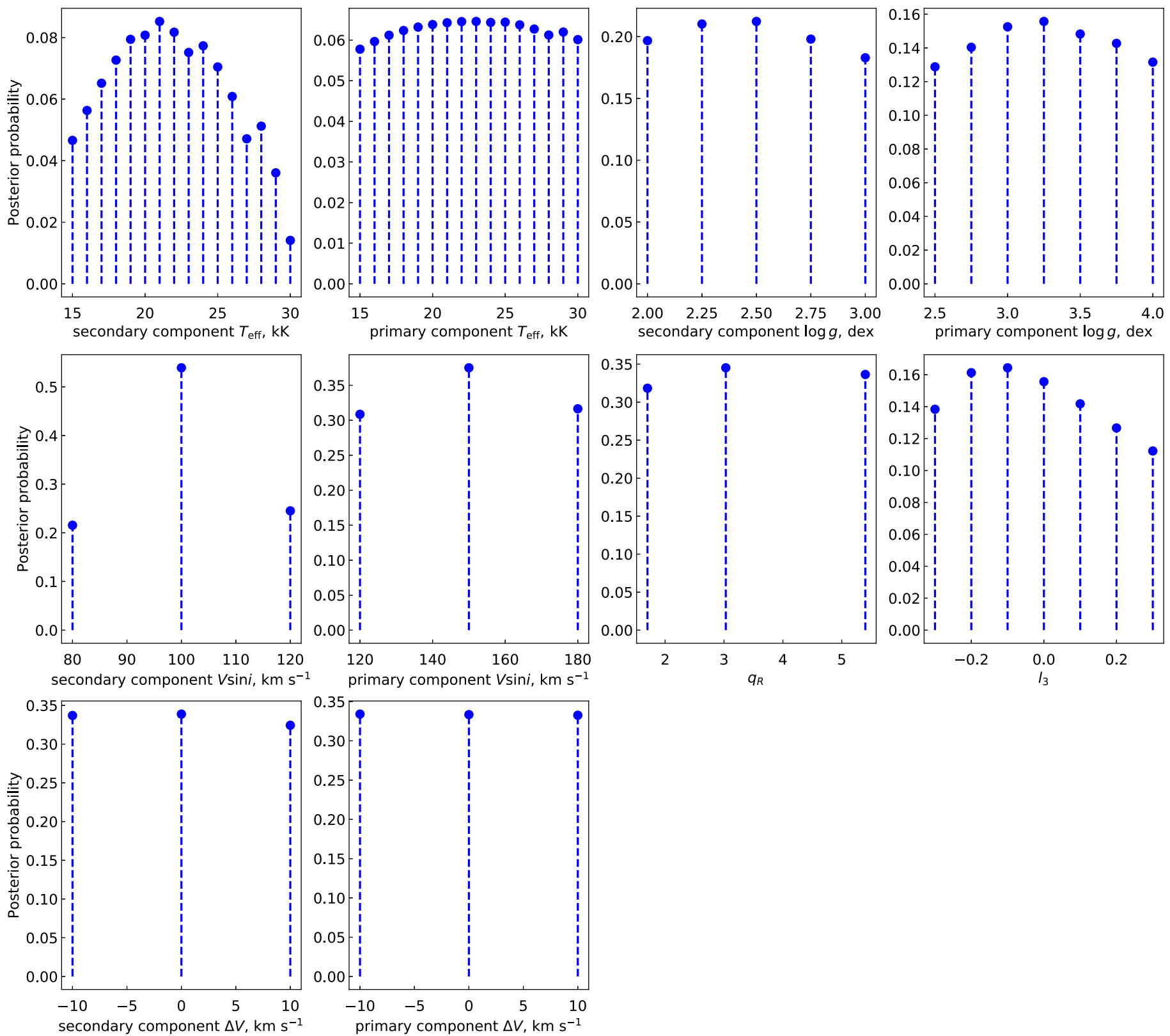

Figure 1. Marginalized posterior probabilities estimated for the observed UU Cas spectrum obtained on 2018 January 5 (orbital phase of 0.2738 ).

The theoretical spectrum with parameters estimated for the orbital phase of 0.2738 fits the Balmer and metal lines in the majority of the observed spectra obtained at other phases. However, the profiles of the hydrogen and helium lines in some of the observed spectra have a notable absorption feature (see Figure 4 at velocities of $\sim-200 \mathrm{~km} \mathrm{~s}^{-1}$ ). The feature is seen in the spectra observed at phases of $0.0838,0.1378,0.2268,0.2617$, 0.3404 , and 0.3756 . We were unable to obtain the synthetic spectra that fit all of the line profiles, including the absorption feature at these phases. For example, for the observed spectrum obtained on 2018 January 12 (orbital phase of 0.0838), where the absorption feature is most prominent, the maximum marginalized posterior probability was obtained for the model parameters given in Table 4. The synthetic spectrum calculated with these parameters does not fit well the observed spectrum (see Figure 4). On the other hand, the theoretical spectrum calculated with the parameters estimated for the orbital phase of 0.2738 fits the Balmer lines profiles observed at an orbital phase of 0.0838 , with the exception of the absorption feature. The same is true for spectra at other phases where the feature is apparent. This feature is also seen in the $\mathrm{H} \alpha$ emission profiles. We suggest that this absorption feature is related to the circumstellar gas, which we discuss further in Section 4. This additional absorption also can be responsible for the fact that the third light contribution $l_{3}$ tends to negative values (see Figure 1), meaning that observed $\mathrm{H}$ and $\mathrm{He}$ lines, which have significant contributions to the $P(D \mid \Theta)$ values, are deeper than expected from the stellar models. The tendency of $l_{3}$ to produce negative values can also be related to uncertainties in chemical composition and $v_{t}$.

As the parameters estimated for the orbital phase of 0.2738 (see Table 3) allow us to fit the spectra at other phases, we used them to construct the theoretical $\mathrm{H} \alpha$ photospheric absorption 

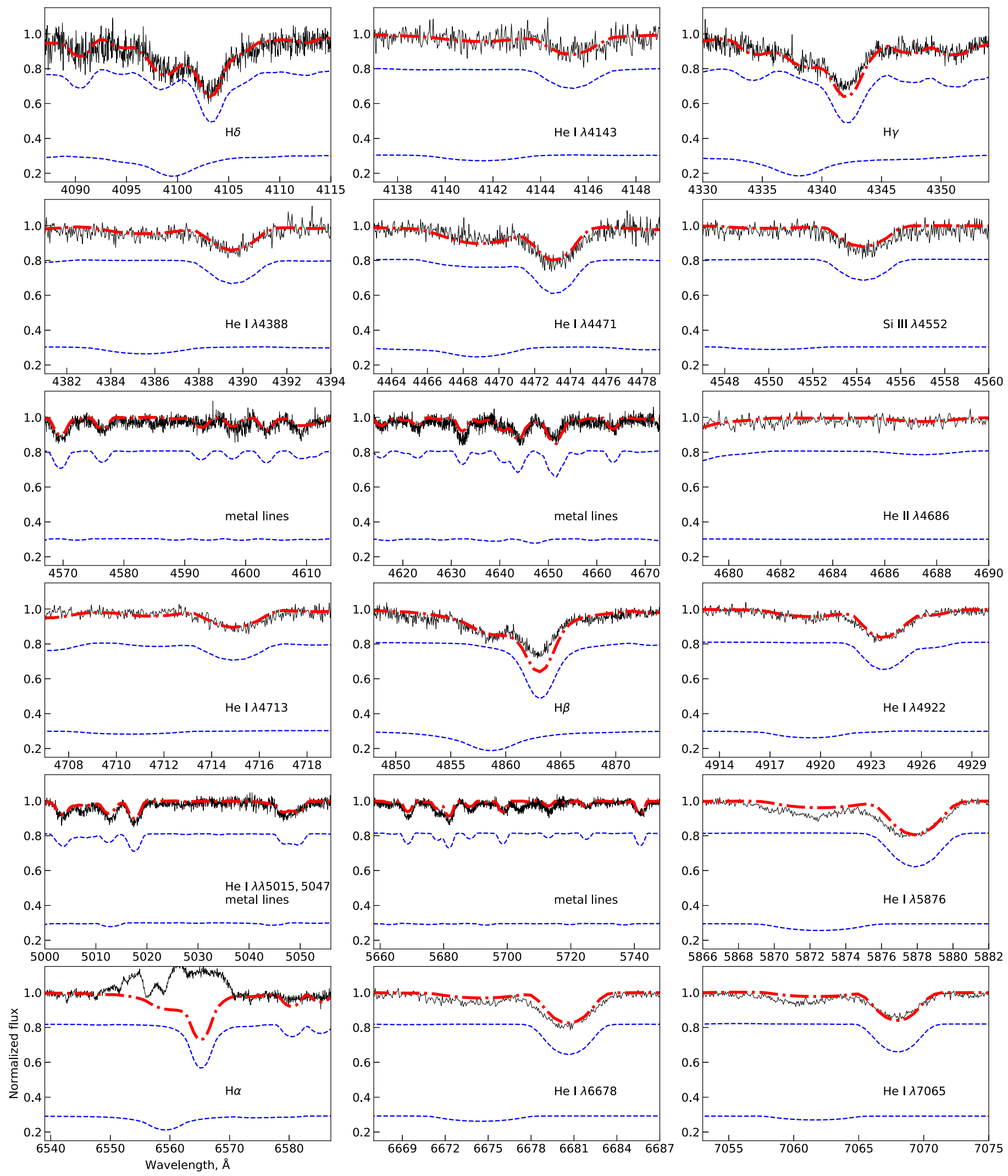

Figure 2. The observed UU Cas spectrum obtained on 2018 January 5 (orbital phase of 0.2738 , black solid line), total theoretical spectrum of the binary (red dasheddotted line), and theoretical spectra of the primary (lowest blue dashed line) and secondary (highest blue dashed line) calculated for the parameters given in Table 3 . The contribution of the third light is not shown.

profile and subtract it from the observed spectra at all phases. The exception is that we set $\Delta V_{1}=0 \mathrm{~km} \mathrm{~s}^{-1}$ as a more realistic estimate, and we account for the fact that the velocity shift of
$10 \mathrm{~km} \mathrm{~s}^{-1}$ of the primary's spectrum does not significantly affect the Balmer line profiles due to the relatively small contribution of the primary's flux in the the total binary spectrum. 
Table 3

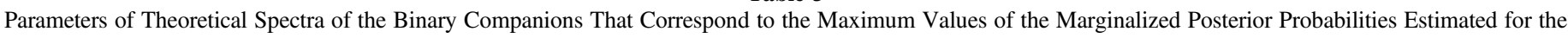
Observed UU Cas Spectrum Obtained on 2018 January 5 (Orbital Phase of 0.2738)

\begin{tabular}{|c|c|c|c|c|c|c|c|c|}
\hline Companion & $\begin{array}{l}T_{\text {eff }} \\
(\mathrm{K})\end{array}$ & $\log g$ & $\begin{array}{c}V \sin i \\
\left(\mathrm{~km} \mathrm{~s}^{-1}\right)\end{array}$ & $\begin{array}{c}v_{t} \\
\left(\mathrm{~km} \mathrm{~s}^{-1}\right)\end{array}$ & Chem. Composition & $\begin{array}{c}\Delta V \\
\left(\mathrm{~km} \mathrm{~s}^{-1}\right)\end{array}$ & $q_{R}$ & $l_{3}$ \\
\hline Primary & 23,000 & 3.25 & 150 & 2 & solar & -10 & 3 & -0.1 \\
\hline Secondary & 21,000 & 2.5 & 100 & 10 & supergiant & 0 & & \\
\hline
\end{tabular}
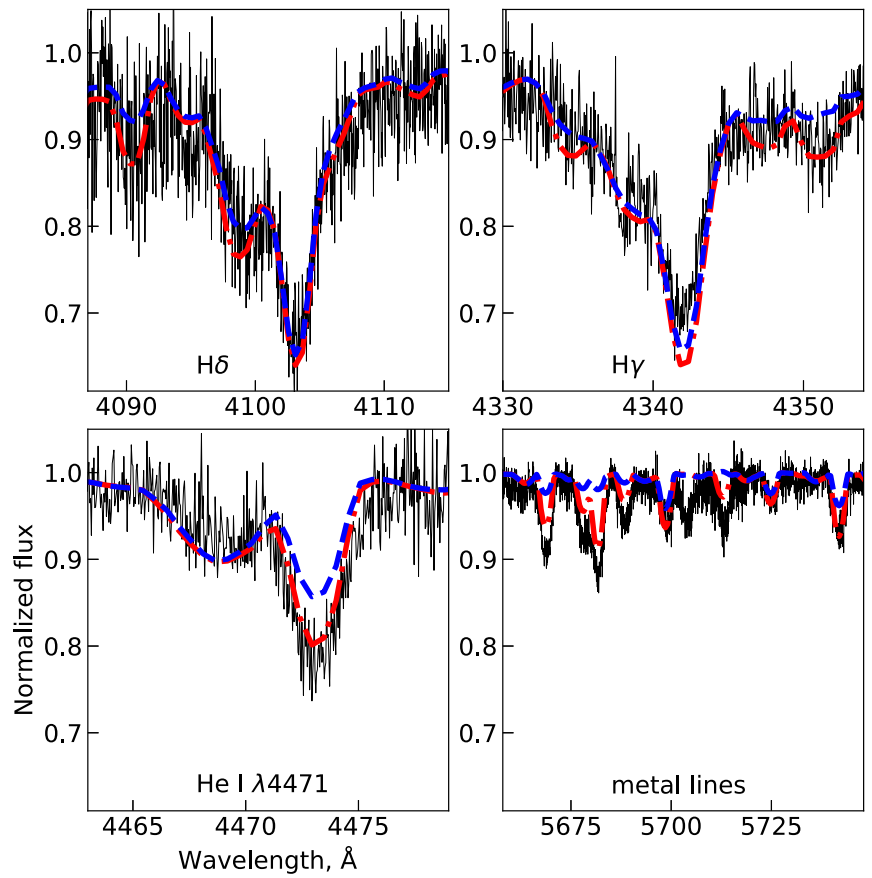

Figure 3. Shown here are the observed UU Cas spectrum obtained on 2018 January 5 (orbital phase of 0.2738 , black solid line), the theoretical spectrum of the binary calculated for parameters from Table 3 (red dashed-dotted line), and the theoretical spectrum of the binary calculated for the parameters from Table 3, except that the spectrum for the secondary was calculated for the solar chemical composition and $v_{t}=2 \mathrm{~km} \mathrm{~s}^{-1}$ (blue dashed line). The contribution of the third light is not shown.

The range of possible values of $\log g$ for the secondary indicates that this companion can be a giant or supergiant. The primary companion likely has higher values $\log g$ and can be a giant or dwarf. Our analysis also shows that the primary can be relatively hot with $T_{\text {eff }} \sim 30,000 \mathrm{~K}$. Such a hot and massive star can produce wind, which can affect the profile of $\mathrm{H} \alpha$ line. The wind parameters are usually estimated by fitting the $\mathrm{H} \alpha$ and $\mathrm{H} \beta$ line profiles with the synthetic spectra. We cannot estimate the wind parameters, as the emission in the $\mathrm{H} \alpha$ and $\mathrm{H} \beta$ lines likely traces not only the wind but other circumstellar gas structures as well (see Section 4). Their relative contribution in the total emission is not known. Ultraviolet spectroscopic observations can aid the estimation of the stellar wind parameters (see, e.g., Martins 2011), which we recommend as a subject for future study. Moreover, as was noted in the Introduction, the higher temperature of the primary contradicts the fact that the deeper light-curve minimum corresponds to the eclipse of the secondary. This contradiction can be resolved by assuming the presence of an accretion disk around the primary; however, we do not account for this scenario. On the other hand, as the primary's $T_{\text {eff }}$ posterior probability distribution is practically flat, we cannot exclude the possibility that the primary has a similar or even lower $T_{\text {eff }}$ in comparison to the secondary based on our spectroscopic analysis alone.

\section{Analysis of $\mathrm{H} \alpha$ Profiles and Flow-pattern Model}

To study the flow pattern in the system, we needed to ensure that the emission $\mathrm{H} \alpha$ profiles were free of atmospheric absorptions that, as we believe, ${ }^{7}$ originated in gaseous structures surrounding the companions. In Figure 5, we plot the original $\mathrm{H} \alpha$ profiles (black) and the profiles after the removal of the absorption photospheric components (red).

The resulting profiles reveal a spectral feature that has already been noted in Section 3. This feature is a "residual" absorption component that is prominent within the phase interval from $\phi \approx 0.1$ to $\phi \approx 0.27$. It is clearly visible that this absorption component disappears near $\phi \approx 0.27$, i.e., the line intensity even in the wings does not fall below zero. This behavior of the profiles prompted us to propose a phenomenological model of the flow pattern and line formation, as illustrated in Figure 6.

According to the model, we suppose that a single profile of the observed $\mathrm{H} \alpha$ line consists of at least three major components originating in: the stream from the $L_{1}$ point (red solid line in Figure 6); stellar wind from the hot and massive primary (green solid line in Figure 6); and a disk-like shell surrounding the primary (blue dashed line in Figure 6). The bold black line in Figure 6 represents the resulting profile that is a sum of the red, green, and blue profile components.

To explain the observed behavior of the profiles, we suppose that when looking at the system between the phases $\phi \approx$ 0.0-0.27 (bottom-right corner of the scheme in Figure 6), we see the absorption blue wing of a classical P Cygni profile originating in the stellar wind from the primary. It is not yet saturated by the emissions from the stream and disk, since:

1. at these orbital phases, we look through the stream flowing away from the observer, which shifts its spectral contribution toward the red wing of the resulting profile (red solid line, bottom-right corner of the scheme in Figure 6)

2. the gas velocity at the innermost edge of the disk is higher than the velocity of the P Cygni blue absorption wing, which makes the disk's double-peaked profile ${ }^{8}$ broader and, hence, shifts the blue peak (blue dashed line, bottom-right corner of the scheme in Figure 6) farther toward the high-frequency part of the resulting line profile. Also, it can clearly be seen in Figure 5 that as one moves toward higher frequencies, the absorption again turns into emission.

When we look at the system within the phase interval $\phi \approx 0.5-0.9$ (top-left corner of the scheme in Figure 6), the stream is directed to the observer. Thus, its contribution (red solid line, top-left corner of the scheme in Figure 6) shifts toward the

\footnotetext{
7 Which we provide proof for below.

8 It is well known that the profiles of emission lines originating in accretion disks are double-peaked.
} 
Table 4

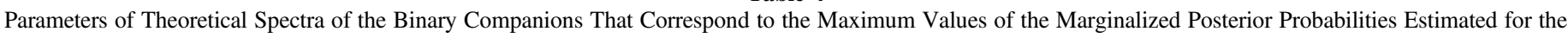
Observed UU Cas Spectrum Obtained on 2018 January 12 (Orbital Phase of 0.0838)

\begin{tabular}{|c|c|c|c|c|c|c|c|c|}
\hline Companion & $\begin{array}{l}T_{\text {eff }} \\
(\mathrm{K})\end{array}$ & $\log g$ & $\begin{array}{c}V \sin i \\
\left(\mathrm{~km} \mathrm{~s}^{-1}\right)\end{array}$ & $\left(\begin{array}{c}v_{t} \\
\left(\mathrm{~km} \mathrm{~s}^{-1}\right)\end{array}\right.$ & Chem. Composition & $\begin{array}{c}\Delta V \\
\left(\mathrm{~km} \mathrm{~s}^{-1}\right)\end{array}$ & $q_{R}$ & $l_{3}$ \\
\hline Primary & 25,000 & 3.5 & 150 & 2 & solar & -10 & 3 & -0.3 \\
\hline Secondary & 21,000 & 3.0 & 100 & 10 & supergiant & -10 & & \\
\hline
\end{tabular}
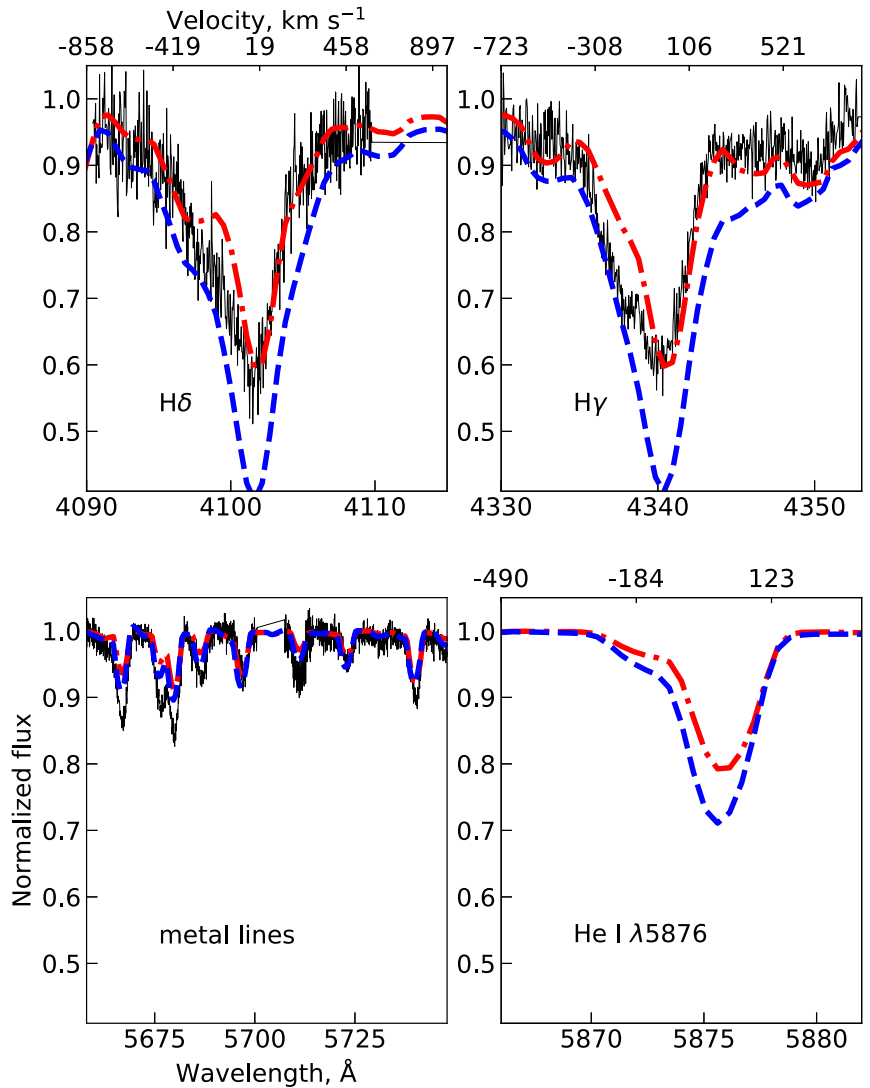

Figure 4. Shown here are the observed UU Cas spectrum obtained on 2018 January 12 (orbital phase of 0.0838 , black solid line), the theoretical spectrum of the binary calculated for the parameters from Table 3 (red dashed-dotted line), and the theoretical spectrum of the binary calculated for the parameters from Table 4 (blue dashed line). The contribution of the third light is not shown.

blue wing of the resulting profile and saturates the absorption wing of the P Cygni line component originating in the primary's stellar wind. Note that, in Figure 5, prominent absorption features disappear after the phases $\phi=0.274-0.368$, since the projection of the stream velocity onto the line of sight at phases close to $\phi=0.5$ and beyond becomes negative, which shifts the spectral contribution of the stream toward the blue.

\section{H $\alpha$ Doppler Tomography}

To examine our phenomenological model of the flow pattern, we use Doppler tomography (Marsh \& Horne 1988). This technique allows us to convert observed emission line profiles into specific intensity maps. In many cases, these maps (hereafter tomograms) are more illustrative than the initial line profiles. The fact is that the Doppler tomogram represents a gas dynamical pattern in the close binary system in the velocity space $\left(V_{x}, V_{y}\right.$; orbital plane). Since its invention in 1988 , this method has been used in a great number of works and helped reveal several gas dynamic elements common for close semidetached binary systems, e.g., the accretion disk and the stream from the $L_{1}$ point. Therefore, now, in most cases, we can confidently identify these particular elements on the tomogram (if they exist in the object) and use tomography to test relatively simple models (such as that proposed in Section 4).

The initial data for the UU Cas Doppler tomogram are the profiles shown in Figure 5 in red. In some of these spectra, we had to set the negative values (below the level of continuum) to zero, since Doppler tomography does not work with absorptions. This trick, of course, violates one of the basic assumptions of Doppler tomography, implying that the flow pattern must be absolutely transparent for the radiation in the given line. However, at this step, we unfortunately cannot adequately separate the contributions of different gas dynamic elements and pick out only the line components originating in transparent structures. Thus, we can only assume the circumstellar gas contribution to be optically thin. In addition, looking ahead, we can say that, in this particular case, setting negative values to zero does not significantly influence our results. The computational code we used to construct the tomogram is based on the Lucy (1994) algorithm with Maximum Entropy Regularization.

The resulting tomogram is shown in Figure 7. The figureeight-like shape in black represents the Roche lobes of the companions (lower half-primary, upper half-secondary). In the tomogram, one can see three main features. The brightest spot that is mostly enclosed within the Roche lobe of the secondary we associate with the region of secondary's surface facing the primary companion and irradiated by its radiation. Analogous spots on the donor's surface facing the hot accretor have been reported in a number of papers devoted to cataclysmic variables (see, e.g., Steeghs et al. 2001; Steeghs 2004; Kononov et al. 2008). The extended bright structure stretching toward the lowerleft quadrant of the tomogram can be confidently associated with the stream from the $L_{1}$ point. The shape and broadness of the stream indicate that it may not be as collimated as the streams in the cataclysmic variables. The tomogram shows that material apparently escapes from a broader area of secondary's surface. In addition, considering the tomogram, where the bulk of emission appears to be enclosed within the secondary's Roche lobe, we, probably, should attribute the spectral feature shown in Figure 6 in red to both the stream and secondary's surface facing the accretor.

There is also a weak disk-like structure that is centered at the point with the coordinates of $V_{x} \approx 0 \mathrm{~km} \mathrm{~s}^{-1}$ and $V_{y} \approx$ $-100 \mathrm{~km} \mathrm{~s}^{-1}$ and having a "velocity" radius of $R_{V} \approx$ $500-600 \mathrm{~km} \mathrm{~s}^{-1}$. To make its position and limiting velocities clearer, we have enclosed this diffuse feature by the dashed 


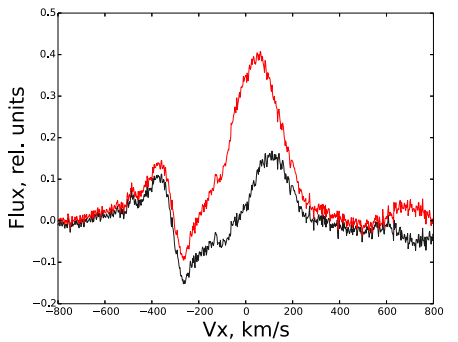

$\phi=0.084$

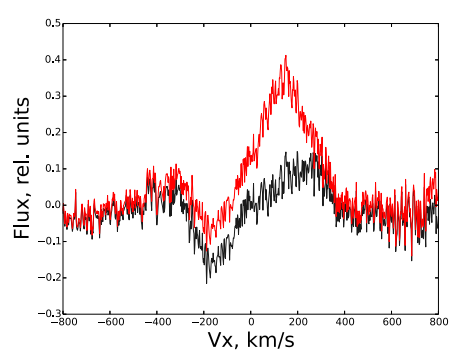

$\phi=0.223$

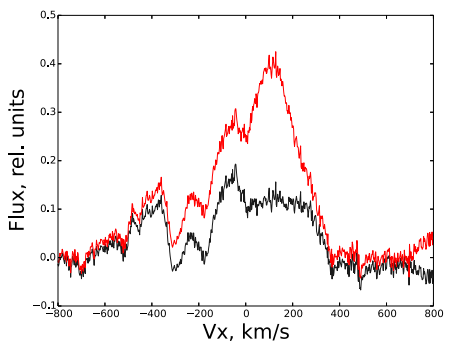

$\phi=0.274$

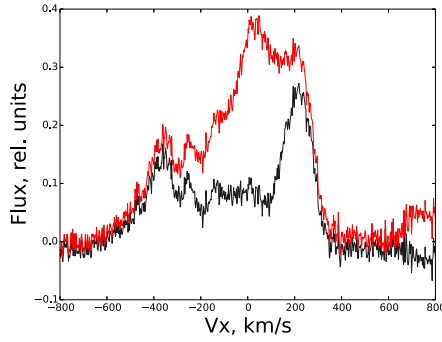

$\phi=0.38$

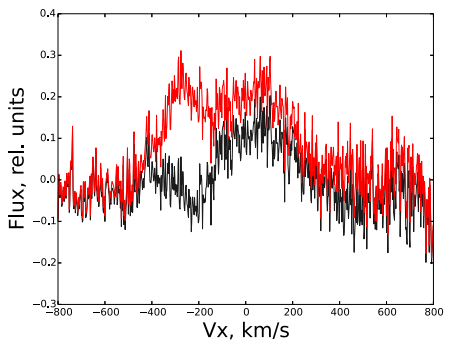

$\phi=0.772$

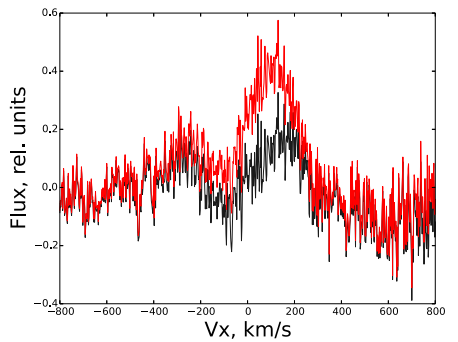

$\phi=0.138$

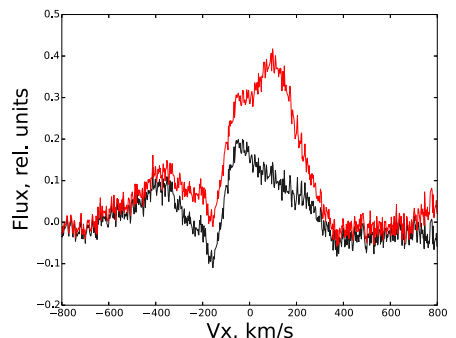

$\phi=0.261$

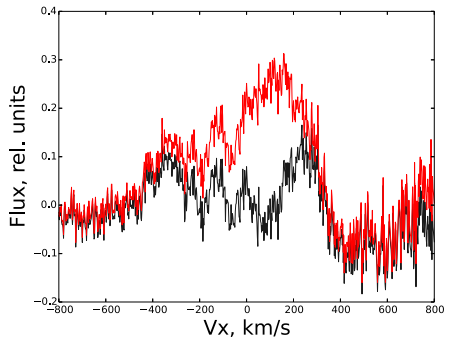

$\phi=0.34$

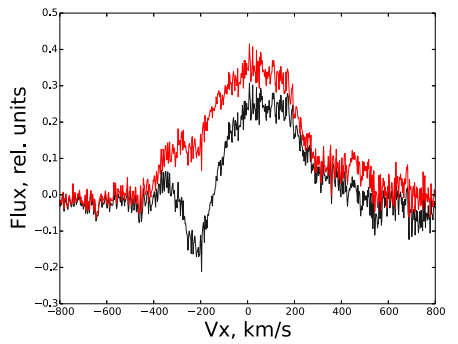

$\phi=0.636$

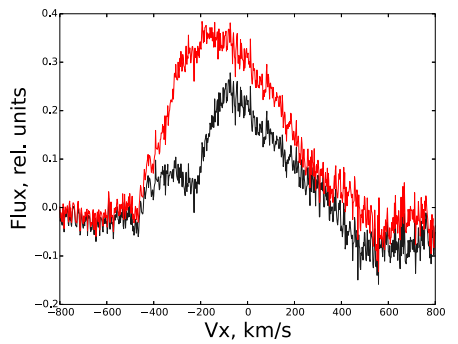

$\phi=0.812$
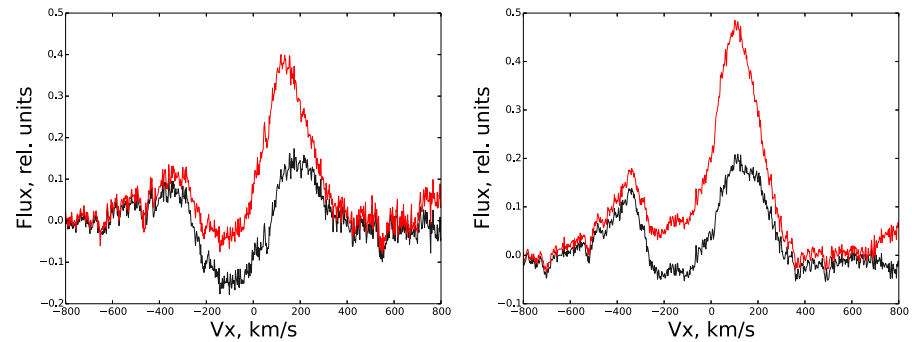

$\phi=0.166$

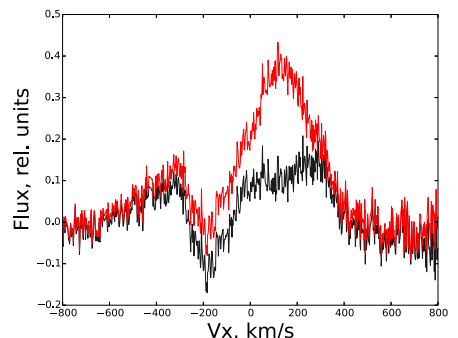

$\phi=0.262$

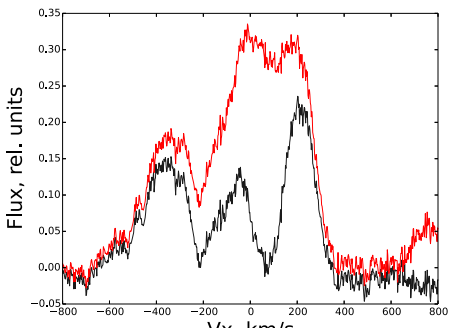

$\phi=0.368$

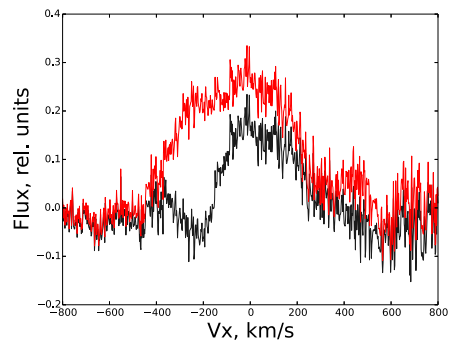

$\phi=0.673$

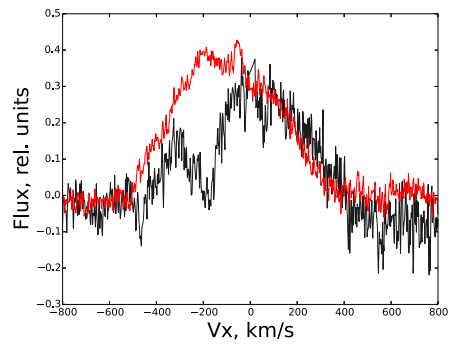

$\phi=0.833$ $\phi=0.191$

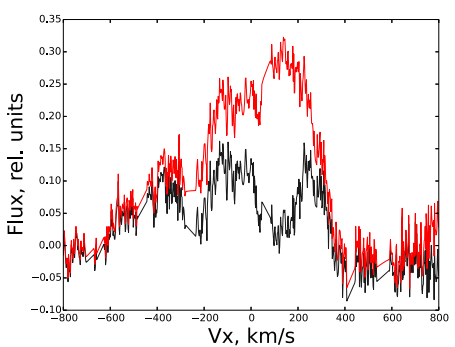

$\phi=0.264$

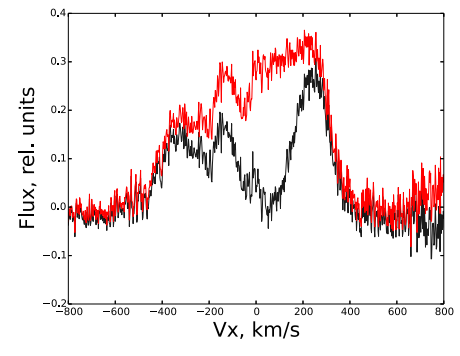

$\phi=0.376$

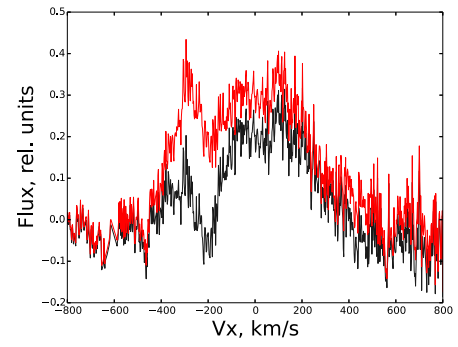

$\phi=0.72$

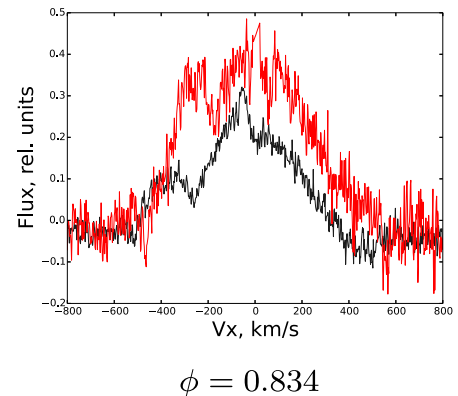

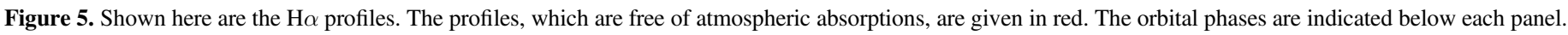

circle in the tomogram. Note that the position of the disk center supports the findings of Gorda (2017) and of this paper; namely, the estimated amplitude of the primary's radial velocity $V_{\mathrm{r} 1} \approx 100 \mathrm{~km} \mathrm{~s}^{-1}$. This allows us to attribute the disk-like structure to the primary companion.
As an additional support of our findings, we should mention the paper by Richards et al. (1995) where authors studied Algol-type binaries and reported many of the same tomogram features that we do: streams, contributions from secondary companions, and disk-like structures. 

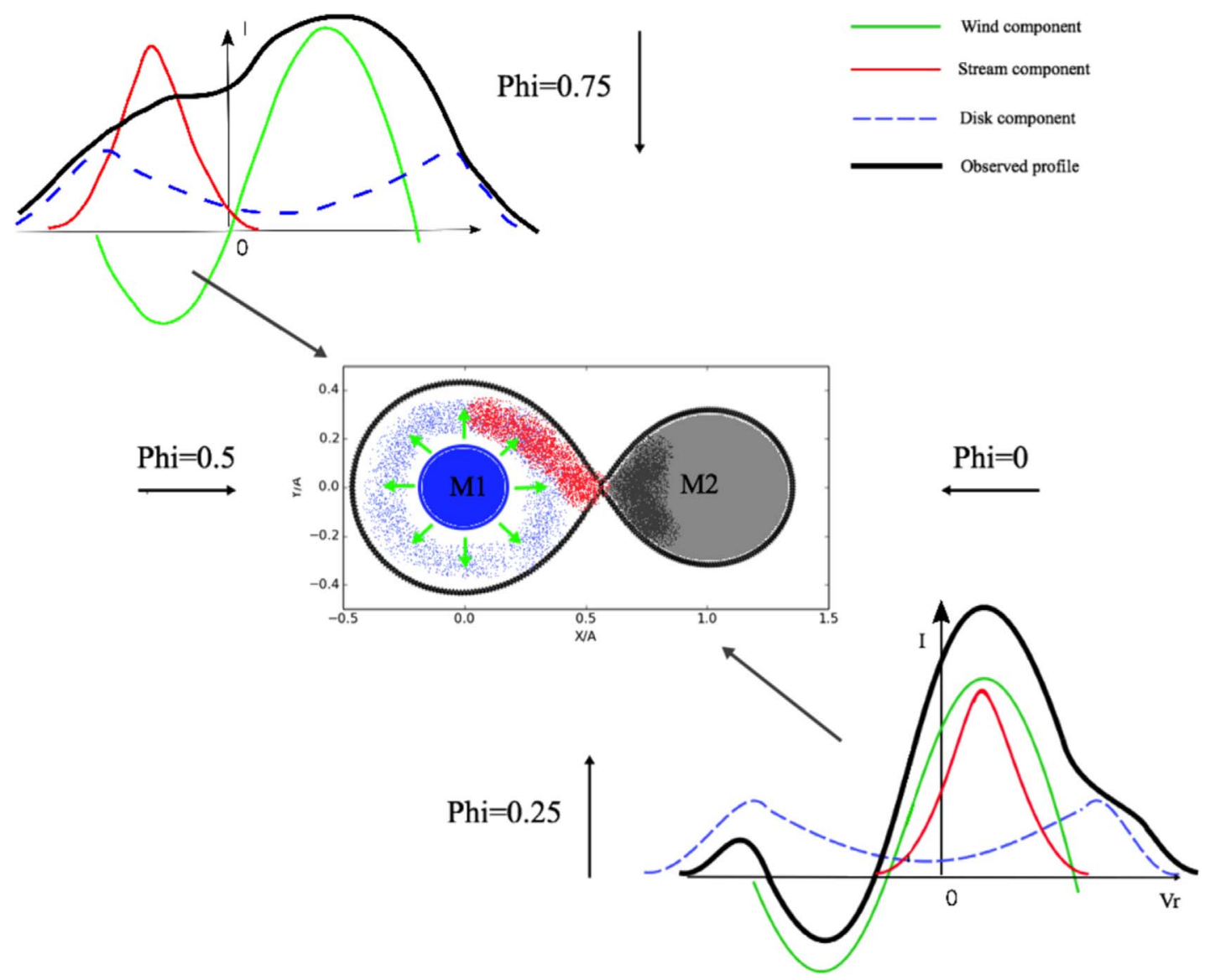

Figure 6. A schematic view of gas dynamic features in the UU Cas and their possible observational manifestations. The green solid lines in the profiles (top-left and lower-right corners of the scheme) represent the P Cygni component originating in the stellar wind from the primary. The red solid lines represent the contribution of the stream from the $L_{1}$ point. The blue dashed lines are the profile component originating in the disk. The black bold lines are the resulting observed profiles (compare with Figure 5). Corresponding gas dynamic structures are shown in the center of the scheme in the same colors. The orbital phases in the notation accepted in this paper are also shown.

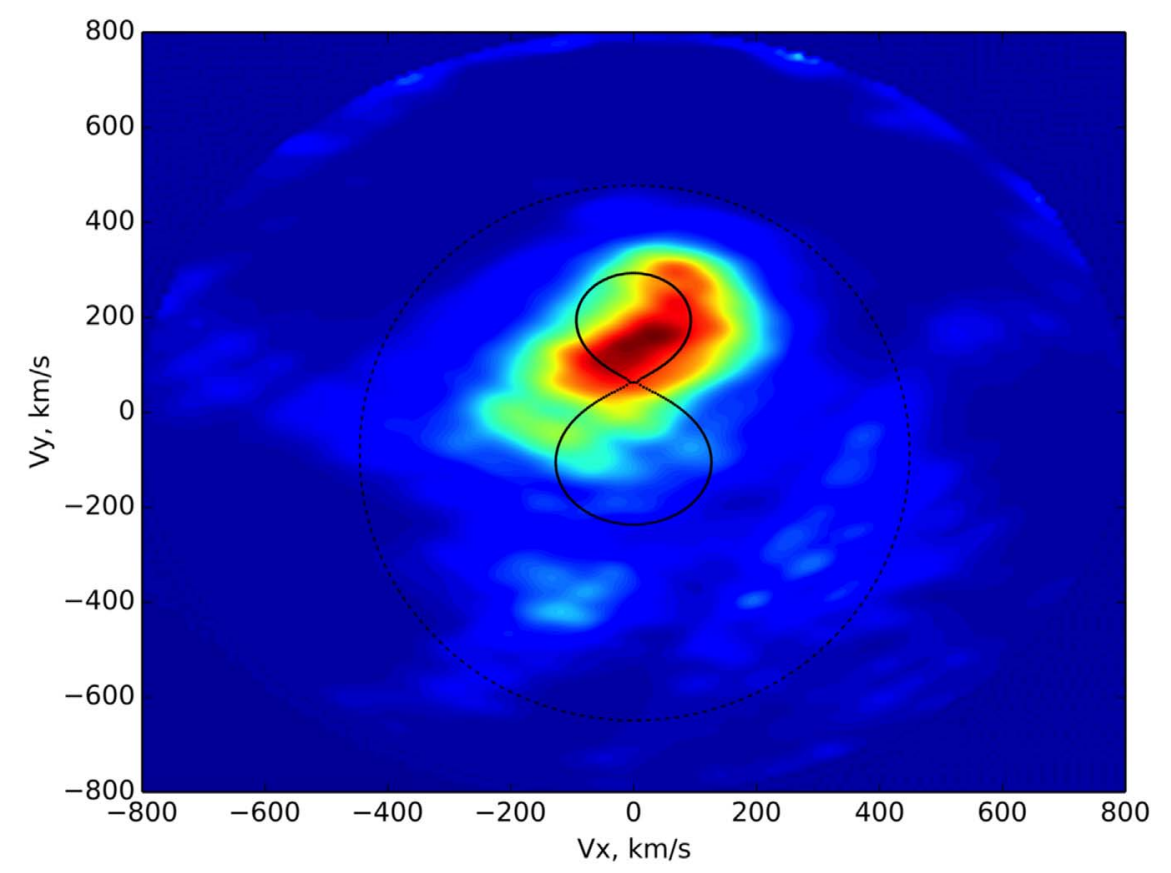

Figure 7. Shown here is the H $\alpha$ Doppler tomogram of UU Cas. The figure-eight-like shape in black represents the Roche lobes of the companions. The secondary's Roche lobe is within positive $V_{y}$ velocities. The dashed circle centered on the orbital velocity of the primary encloses a diffuse feature that, as we suppose, is produced by the radiation of a disk-like structure surrounding the massive companion. 


\section{Estimates of Stellar Radii and Luminosities}

To examine the finding of Sections 4 and 5, we have obtained some quantitative estimates of the companions' radii and the Keplerian velocity at the inner radius of the accretion disk that may surround the primary. The stellar parameters estimated in Section 3 are uncertain; therefore, we made an additional assumption to estimate the stellar radii. Based on the assumption that the secondary fills its Roche lobe (as follows from Sections 4 and 5), we can limit its size by using the equation for the volume radius of its Roche lobe given by Paczyński (1971)

$$
\frac{R_{L 2}}{a}=0.38+0.2 \log q,
$$

where $R_{L 2}$ is the volume radius of the secondary's Roche lobe, $a$ is the binary separation in solar radii, and $q=M_{2} / M_{1}$ is the mass ratio of the companions. Substituting the values of $a=52.7 \pm 0.5 R_{\odot}$ and $q=0.54 \pm 0.01$ (Gorda 2017) into the above equation yields the value $R_{2}=R_{L 2}=17.2 \pm 0.3 R_{\odot}$. To estimate other parameters, we used a Monte-Carlo method with the number of samples of $10^{6}$. Hereafter, we report the median values of a given parameter distribution and the errors corresponding to 32th and 68th percentiles of this distribution. The primary's radius $R_{1}=9.9_{-2}^{+0.5} R_{\odot}$ was estimated by sampling the distribution of $q_{R}$ obtained for the orbital phase of 0.2738 in Section 3 and normal distribution of $R_{2}$ with a mean and standard deviation of $17.2 R_{\odot}$ and $0.3 R_{\odot}$, respectively. Sampling the primary's mass from the normal distribution with a mean and standard deviation of, respectively, $17.7 M_{\odot}$ and $0.2 M_{\odot}$ (Gorda 2017), and $R_{1}$ distribution, we obtain the Keplerian velocity at the primary's radius $V_{K}=580_{-80}^{+40} \mathrm{~km} \mathrm{~s}^{-1}$. Using the distribution of $V_{K}$ and the normal distribution of the system's inclination $i$ with a mean of $68^{\circ}$ and standard deviation of $2^{\circ}$ (Polushina 2002), we estimate $V_{K} \sin i=540_{-80}^{+40} \mathrm{~km} \mathrm{~s}^{-1}$, which, given the uncertainties, is in good agreement with the "velocity" radius of the disk-like feature in the tomogram (Figure 7). This last estimate may be considered an additional argument supporting the results of Sections 4 and 5, and it shows that the size of the disk-like feature in the tomogram (Figure 7) is naturally limited by the Keplerian velocity at the primary's surface.

With the Monte-Carlo method, we also estimated the stellar luminosities, which can be useful for the system's evolution studies. Using the distributions of $R_{2}$ and $T_{\text {eff } 2}$, we have estimated the secondary's bolometric luminosity $\log L_{2} / L_{\odot}=4.7 \pm 0.2$. The luminosity of the primary component $\log L_{1} / L_{\odot}=$ $4.4 \pm 0.2$ was estimated in the same manner as that of the secondary component.

\section{Conclusions}

In this paper, we have considered the results of spectral observations of the close interacting binary system UU Cas. We obtained a series of phase-resolved spectra that allowed us to analyze the fundamental parameters and reveal gas dynamic elements that presumably exist in the system.

Using the spectral data, we have estimated the parameters of both of the companions (see Table 3). By considering the orbital evolution of the $\mathrm{H} \alpha$ emission line profiles, we have proposed a phenomenological model of the flow pattern in the system. In particular, we have found the evidence of at least three gas dynamic structures: the stream from the $L_{1}$ point; the disk surrounding the primary companion; and the stellar wind from the primary, whose existence is suggested by the presence of a P Cygni component in the line profiles (see Section 4 and Figure 6). To test our model, we have used the same $\mathrm{H} \alpha$ profiles to compute a Doppler tomogram of UU Cas. By analyzing the tomogram, we can confidently confirm the existence of the stream and disk. According to the position and size of the disk-like structure in the tomogram, we can attribute it to the primary companion.

All of the findings show that UU Cas is a semi-detached system, whose less massive companion fills its Roche lobe and transfers material to the Roche lobe of the massive primary companion. At present, we see no evidence of the common envelope in the system, and neither can we consider it to be a contact system, as outlined by the following reasons:

1. We see the stream from the L1 point directed from the donor to the accretor. And this stream is clearly seen propagating to some extension, which means that it does not immediately collide with the accretor's surface. As the analysis of the emission line profiles and the proposed model show, we see this stream from almost all of the phases, while in a contact system, or a system at the stage of common envelope, this particular form of stream does not exist, in principle.

2. The disk-like structure in the tomogram is centered on the accretor. It is hard to imagine the same picture in a contact system where the companions touch each other and, hence, no disk around a single component can exist.

3. The secondary is less massive, cooler, and brighter, which indicate its much larger surface. Meanwhile, if the more massive and hotter star had been even just slightly close to its Roche lobe, we would have expected it to be brighter.

4. The lines of the accretor demonstrate its faster rotation, which may also suggest that this companion is not synchronized and does not fill its Roche lobe.

In combination with the estimates of the companions' masses (Gorda 2017), our findings allow us to suppose that the system is at the late stage of the first mass transfer phase of its evolution. The fact that the secondary's spectrum is better fitted with the supergiant chemical composition may also hint that the mass transfer started after hydrogen burning had ceased in the core of the current secondary (e.g., case B/case C in the paper by Podsiadlowski et al. 1992 or the Stage 2 discussed by Postnov \& Yungelson 2014, Section 4.1). To make more detailed conclusions on the evolutionary state and further evolutionary path of UU Cas, we need additional high-quality spectral data to investigate the fundamental parameters of both companions.

This paper is part of an ongoing project, and we hope that more detailed information on UU Cas will become available as more data is amassed and analyzed.

G.S. and P.S. acknowledge support by the Ministry of Science (the basic part of the State assignment, RK No. AAAA-A17-117030310283-7) and by the Act No. 211 of the Government of the Russian Federation, agreement No 02. A03.21.0006. 
D.K. appreciates the valuable advice and comments on the evolutionary aspects given by Dr. Lev Yungelson.

Software: DECH (http://www.gazinur.com/DECH-software. html), ROTIN3, TLUSTY (Hubeny \& Lanz 1995), BONNSAI (Schneider et al. 2014).

\section{ORCID iDs}

Dmitry A. Kononov (iD https://orcid.org/0000-00019706-1747

\section{References}

Antokhina, E. A., \& Kumsiashvili, M. I. 1992, SvA, 36, 25

Djurašević, G. R., Vince, I., \& Atanacković, O. 2010, in ASP Conf. Ser. 435,

Binaries-Key to Comprehension of the Universe, ed. A. Prša \& M. Zejda (San Francisco, CA: ASP), 301

Gorda, S. Yu 2017, AstBu, 72, 321

Grevesse, N., \& Sauval, A. J. 1998, SSRv, 85, 161

Hubeny, I., \& Lanz, T. 1995, ApJ, 439, 875

Kononov, D. A., Kaigorodov, P. V., Bisikalo, D. V., et al. 2008, ARep, 52, 835

Krushinsky, V. V., Popov, A. A., \& Punanova, A. F. 2014, AstBu, 69, 497

Kumsiashvili, M. I., \& Chargeishvili, K. B. 2009, arXiv:0907.1047
Lanz, T., \& Hubeny, I. 2007, ApJS, 169, 83

Lucy, L. B. 1994, A\&A, 289, 983

Markov, H., Markova, N., Vince, I., \& Jurasevich, G. 2011, BlgAJ, 15, 87

Markov, H., Vince, I., Markova, N., \& Djurasevic, G. 2010, POBeo, 90 , 159

Marsh, T. R., \& Horne, K. 1988, MNRAS, 235, 269

Martin, N. 1972, A\&A, 17, 253

Martins, F. 2011, BSRSL, 80, 29

Nieva, M.-F., \& Przybilla, N. 2010, in EAS Publ. Ser. 43, Non-LTE Line Formation for Trace Elements in Stellar Atmospheres, ed. R. Monier et al. (Les Ulis: EDP Sciences), 167

Paczyński, B. 1971, ARA\&A, 9, 183

Panchuk, V. E., Yushkin, M. V., \& Yakopov, M. V. 2011, AstBu, 66, 355

Podsiadlowski, P., Joss, P. C., \& Hsu, J. J. L. 1992, ApJ, 391, 246

Polushina, T. S. 2002, ARep, 46, 900

Postnov, K. A., \& Yungelson, L. R. 2014, LRR, 17, 3

Richards, M. T., Albright, G. E., \& Bowles, L. M. 1995, ApJL, 438, L103 Sanford, R. F. 1934, ApJ, 79, 84

Schneider, F. R. N., Langer, N., de Koter, A., et al. 2014, A\&A, 570, A66

Schwarz, G. J., Ness, J.-U., Osborne, J. P., et al. 2011, ApJS, 197, 31

Steeghs, D. 2004, RMxAC, 20, 178

Steeghs, D., Marsh, T., Knigge, C., et al. 2001, ApJL, 562, L145

Tkachenko, A. 2015, A\&A, 581, A129

Vogt, F. P. A., Dopita, M. A., Kewley, L. J., et al. 2014, ApJ, 793, 127

Wellstein, S., \& Langer, N. 1999, A\&A, 350, 148 